\title{
Low Power Consuming Arm Exoskeleton Design Using Force Sensitive Resistors
}

\author{
S.Y. Leong, Y.H. Goh*, C.Y.Teoh \\ *Tunku Abdul Rahman University College, Faculty of Engineering and Built Environment \\ Jalan Genting Kelang, 53300 Kuala Lumpur, Malaysia \\ gohyh@acd.tarc.edu.my
}

Keywords: Arm Exoskeleton; Force Sensitive Resistor; Low Power Consuming

\begin{abstract}
Exoskeleton as a wearable robotic machine has a huge potential to be used in medical, industrial, civilian and military field as it can increase the user's strength and endurance. The scope of this paper covers three major engineering fields which are mechanical, electronic and programming engineering. Design of the physical arm exoskeleton structure, actuator controller, and the microcontroller programming are included in this paper. A cost effective liner actuator which is able to generate large torque is used in the design. For the movement capturing part, two low-cost force sensitive resistors which are able to provide enough accuracy required to detect user motion are used. A linear actuator which can provide sufficient thrust and will not consume any power at any stationary position is chosen to drive the exoskeleton and for power saving purpose. The design arm exoskeleton has been tested and it is able to react according to the user arm movement and provide force to assist user in carrying heavy loads.
\end{abstract}

\section{Introduction}

An exoskeleton is a wearable mechatronics system that combines the operator and the machine as one. The exoskeleton will capture the intention for movement of the user and through the use of actuators, will allow the user to perform said movement with increase strength and endurance. Besides, increasing strength for task support, it also enables people with weaken muscle or disability to walk and perform daily life activities. Due to the usefulness of an exoskeleton, the first ever attempt to develop an exoskeleton, namely Hardiman was developed in 1965 by Mosher et.al for the US military used [7]. Even though the first developed exoskeleton was not fully functional due to the limitation of technology, it sparks the industrial interest in this area and it has become the first reference model of all the following exoskeleton design until today for the use in different area such as: medical, construction and disaster assistance [3,9].

Due to the advancement in automation technology, many prototype arm exoskeletons have been developed and different techniques, different controllers to capture the intention of the user's movements have been applied and tested to the arm exoskeleton such as using flex sensors [2] to control the actuator movements with the user's fingers which will limit the number of total actuator used in an exoskeleton. Potentiometer was attached to the joint to detect movements in [10]. Besides, accelerometer has been included in the exoskeleton $[1,2,10]$. All the above mentioned methods required physical movement of the user in order for the sensor to pick up the movement.

Recently, researchers utilized EEG (Electroencephalogram) and EMG (Electromyogram) signals to control the exoskeleton [5,6,8]. EEG signals are captured using electrodes on the scalp to detect electrical activities. This type of device required training for it to learn the different EEG signals for different types of movement from different users. Only then, the EEG controlled exoskeleton can reacts to the movement of user accordingly. EMG signals are captured by placing electrodes on muscles, it detects the skin surface electrical signal. For EMG controlled exoskeleton, the location of each electrode has to be precise in order to get the correct trigger signal to control the exoskeleton $[5,6,8]$.

The organisation of the paper is as follow: Section 2 begins with the physical arm exoskeleton design and the material used. Section 3 shows the controller circuit design to read the sensors input and to control the linear actuator. In section 4 , microcontroller programming flowchart is included to show the control process. Obtained test results are included in Section 5. Section 6 presents the conclusion of this study.

\section{Arm Exoskeleton Design}

Figure 1 shows the arm exoskeleton structure design. The arm exoskeleton design is based on the human arm structure. The design prototype consists of an upper limb and a lower limb with one single degree of freedom situated at the elbow. Two force sensitive resistor sensors are mounted at the lower limb, slots for the fasteners are shown in the Figure 1 as well. Since the linear actuator used can supply a maximum thrust of $750 \mathrm{~N}$, a low cost material which is able to support a weight of $75 \mathrm{~kg}$ is needed in the design. Material chosen are: 1) $1 / 4$ inch thick and 2-inch width aluminium flat bar for the main arm exoskeleton structure, 2) M8 bolts and nuts are used to fasten all the joints and 3$) 3 \mathrm{~cm}^{2}(1.5 \mathrm{~cm} \mathrm{x} 2 \mathrm{~cm})$ industrial-strength hook and loop fasteners which are adjustable and suitable for frequent easy opening and closing is chosen to secure the exoskeleton arm to the user's arm. 


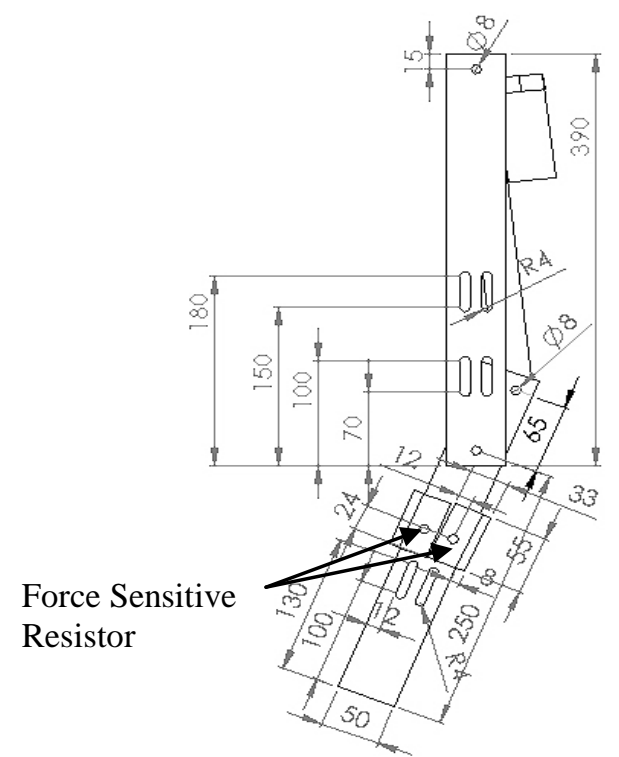

Figure 1: Design of the Exoskeleton Arm

Figure 2 shows the arm exoskeleton backpack. By using the hook and loop fasteners, this backpack supports the arm exoskeleton by distributing the weight and load from the arm exoskeleton to the human body. Besides, it serves as a container for the electrical part of the system such as the battery pack and controller circuit.

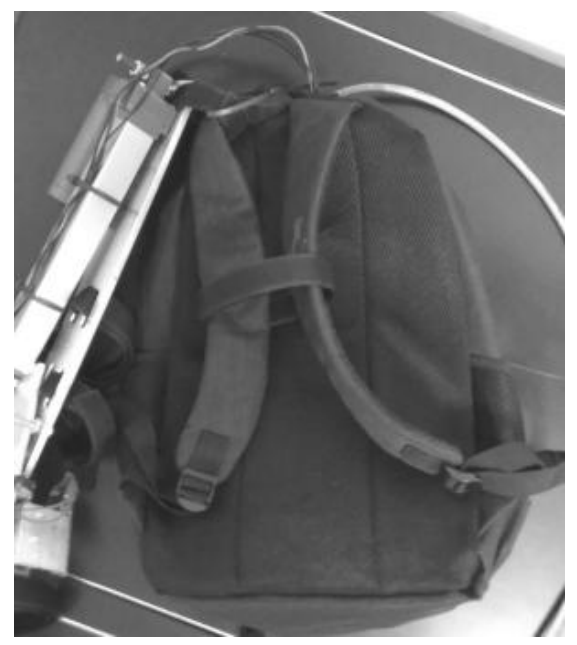

Figure 2: Exoskeleton Arm Backpack

\section{Electrical Circuit Design}

The designed arm exoskeleton will first detects the user's movements through force sensitive resistors and send the signals back to the controller circuit. After processing the detected signal data, controller circuit will control the linear actuator according to the user's arm movement.

\subsection{Microcontroller}

In the designed arm exoskeleton prototype, two analogue input pins for the force sensitive resistors (FSR), a digital input pin for the limit switch, a single pulse width modulation
(PWM) signal output for motor driver speed control and a digital output for the motor direction control are required. Arduino Uno microcontroller has been chosen as it has fourteen digital input and output pins, six analogue input pins and six PWM output pins. Figure 3 shows the electric circuit for the prototype.

To prevent float signal, $10 \mathrm{k} \Omega$ resistors are used as pull down resistors to connect the microcontroller input pins of the FSRs and limit switch to the ground. The limit switch is placed at the inner side of the exoskeleton arm to prevent the arm from further collapsing and fold onto itself which might endanger the user.

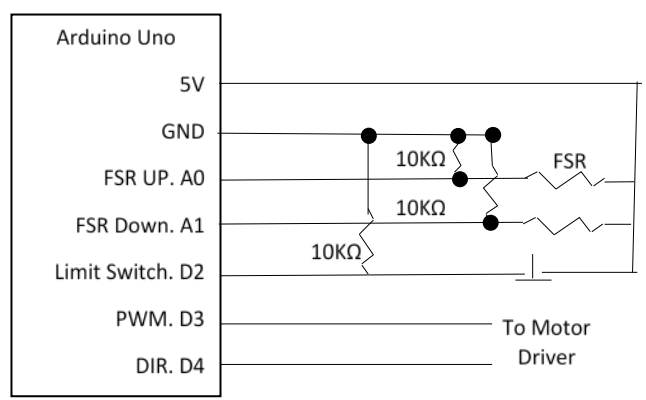

Figure 3: Controller Circuit Design

\subsection{Force Sensitive Resistor}

The FSR sensor is able to detect the magnitude of the force applied. When the apply force is larger, it implies that the will of the user to carry the load is stronger. Controller circuit will sense the change in the force applied and control the speed and force of the linear actuator. Besides, a FSR sensor is cheaper than other types of sensor such as EMG and EEG sensors.

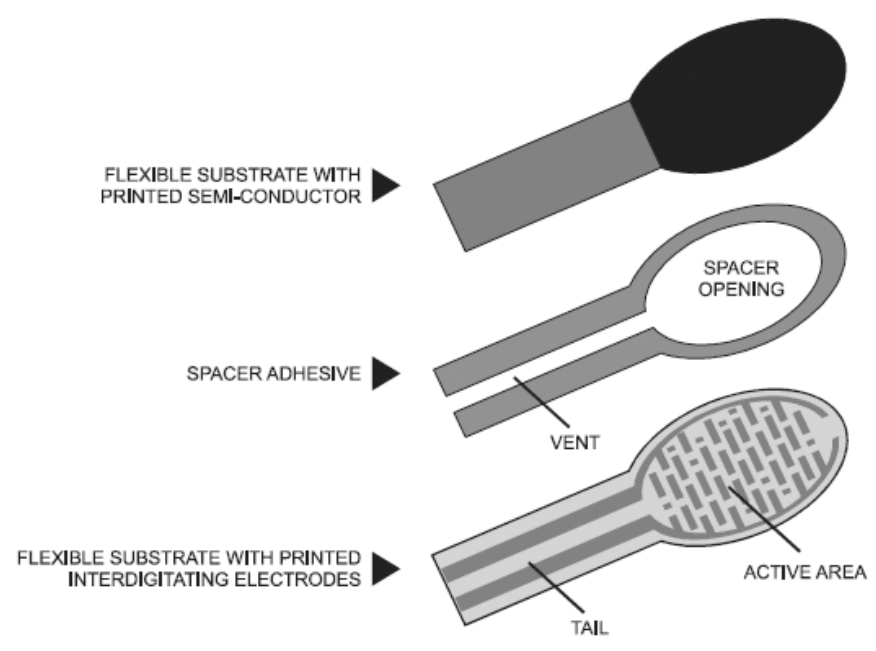

Figure 4: FSR Inner Construction [4]

Figure 4 shows the 3 layers inner construction of a FSR sensor. When force is applied to the active area, it pushes the 
sensing area towards the electrodes. Larger force exerted on the sensing area cause further reduction in the resistance of the force sensitive resistor.

Figure 5 shows the sensor resistance against the force exerted on the sensor. By building a potentiometer using FSR sensor, the change in force will be converted into change in voltage. The controller circuit will read and further process the received signals.

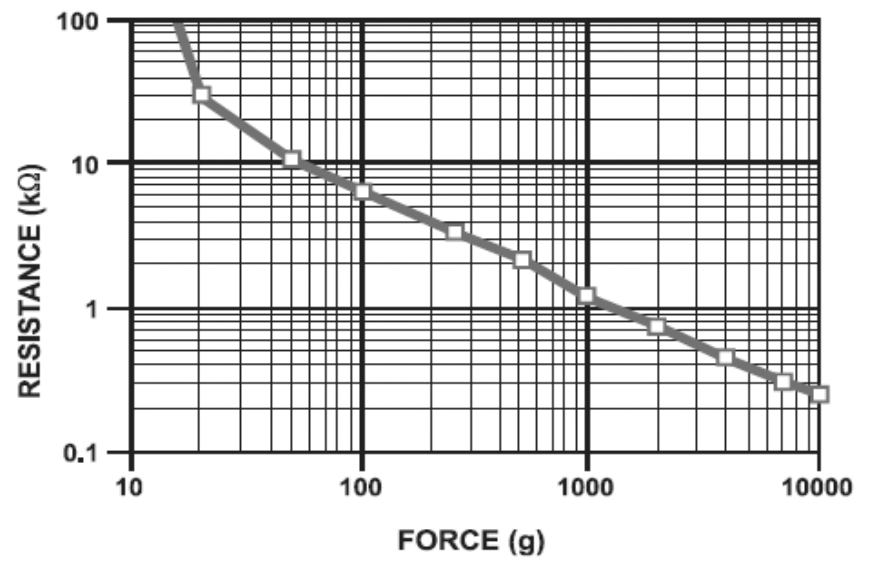

Figure 5: Resistance of FSR sensor against different magnitude of force applied [4].

\subsection{Actuator}

The actuator selected for the exoskeleton arm is a linear actuator. The linear actuator will locked in place when there is no electric power supply to the motor. In this case, the exoskeleton can lift the load, move into position and hold the load in place without consuming any power supply. Besides, if power failure happened, safety of the user carrying heavy load can be ensured since the linear actuator is locked at the stationary position.

Figure 6 shows the dimension of the chosen linear actuator, it operates under $24 \mathrm{~V}$ and can draw up to $3 \mathrm{~A}$ current. It is able to provide a maximum of $750 \mathrm{~N}$ thrust and have an extendable length of $200 \mathrm{~mm}$. The speed of the linear actuator is $20 \mathrm{~mm} / \mathrm{s}$. From the theoretical calculation, the design exoskeleton lever mechanism mounted with the proposed linear actuator can produce a maximum force up to $200 \mathrm{~N}$ in the speed of $77 \mathrm{~mm} / \mathrm{s}$ at the edge of the lower limb.

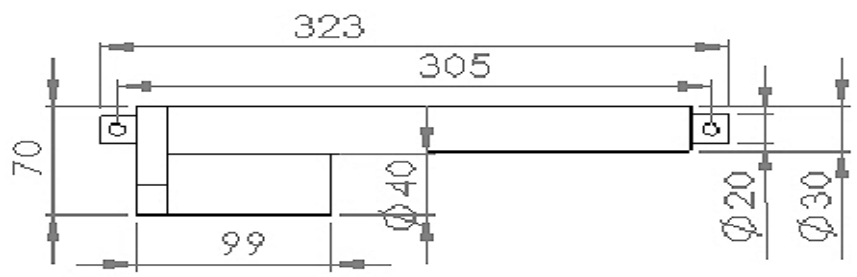

Figure 6: Linear Actuator Drawing

\subsection{Power Supply}

The proposed arm exoskeleton system required two different voltage levels. One is $5 \mathrm{~V}$ for the microcontroller and $24 \mathrm{~V}$ for the motor and the motor driver. In order to reduce clutter, $6 \mathrm{~s}$ Li-Po battery with capacity of $2200 \mathrm{mAh}$ has been used. Voltage regulator is used to step down the $24 \mathrm{~V}$ DC power supply to 5V DC power supply. The chosen battery is able to last for around 45 minutes. However, due to the linear actuator can hold in position without consuming any power supply, the chosen battery can support the proposed arm exoskeleton for longer duration.

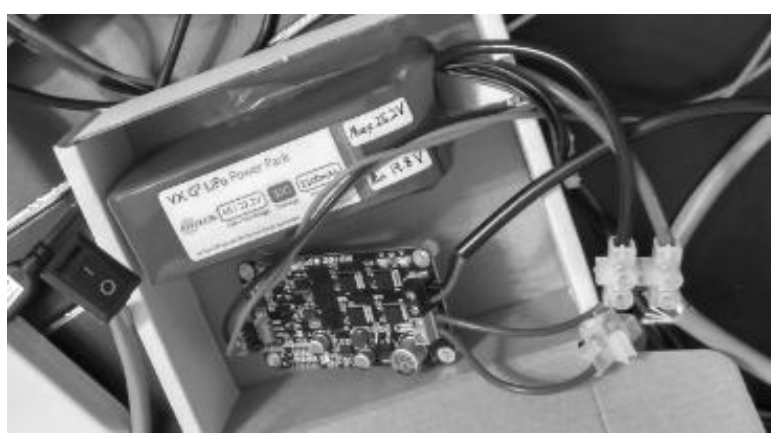

Figure 7: Battery Connection

Figure 7 shows the battery power supply of the proposed system. A switch is installed between the battery and the motor driver for safety purpose.

\section{Microcontroller Programming}

To make the exoskeleton reacts according to the user's intention, the microcontroller takes the data from the FSRs every $10 \mathrm{~ms}$. Noise level has been reduced in the system by averaging every 10 data of the input signals. Difference in the obtained average input values from both FSR sensors will be compared to a preset threshold value. If the difference exceeds the threshold value, the microcontroller will further examine the plus minus sign and the magnitude of the difference in the obtained input values from both FSR sensors. Then, microcontroller sends out the control signal which consists of direction and speed information to the motor driver. The speed control value can be found by dividing the difference of the sensor inputs by 3.3 .

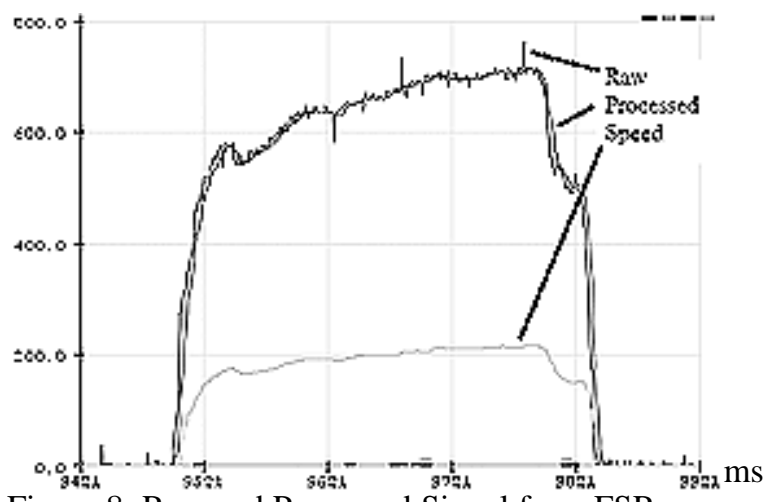

Figure 8: Raw and Processed Signal from FSR 
Figure 8 shows the microcontroller analogue input/ output values ranging from $0-1023$ against time in millisecond. The raw received noisy FSR input signal when force is exerted to the FSR sensor is shown. The noise level has been reduced by averaging every 10 input data. Smooth processed signals can be observed in the same figure. The speed control output signal is obtained by dividing the smooth processed signals by 3.3 .

Figure 9 shows the flow chart of the microcontroller programming algorithm.

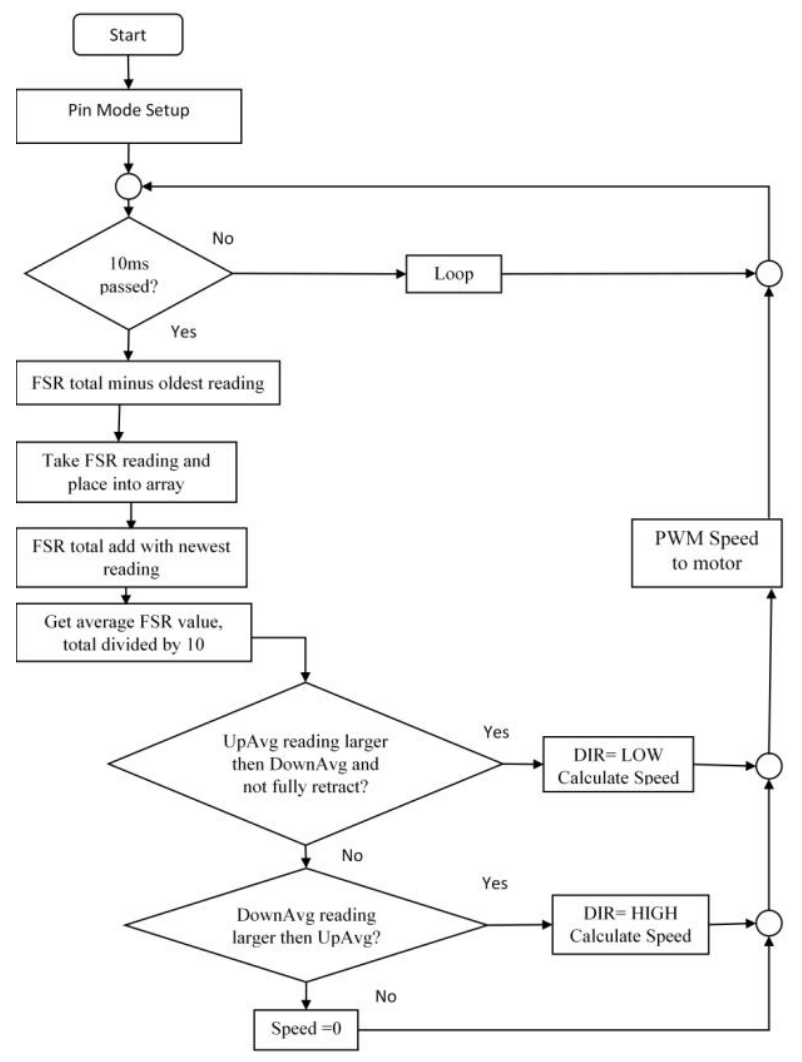

Figure 9: Flow chart of the proposed arm exoskeleton control algorithm.

\section{Result}

Figure 10 shows the built arm exoskeleton prototype. It is able to detect the intention of user and move the actuator in place according to the arm movement. It is able to lift the load up to $15 \mathrm{~kg}$ smoothly. The power consumption is low since battery power is not required while holding the load in place.

\section{Conclusion}

We have developed a low power consumption arm exoskeleton in this paper. It can lift up a $15 \mathrm{~kg}$ weight easily and the low-cost force sensitive sensors provide adequate accuracy for controller system to detect the user motion. It will only draw power from the battery when lifting up or putting down the load. There is no power consumption when holding the load in place.

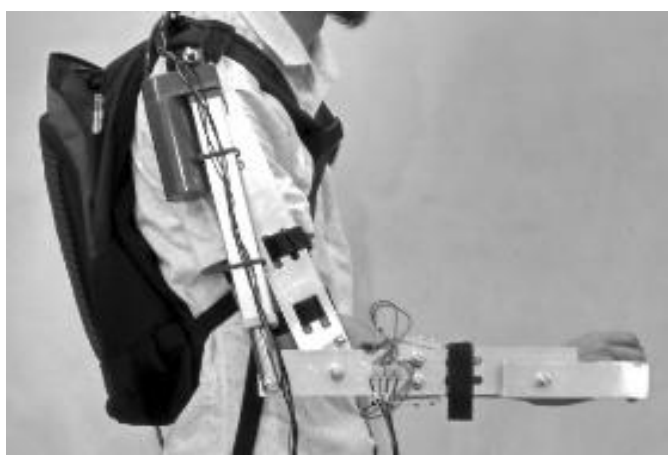

Figure 10: Exoskeleton Arm Prototype

\section{References}

[1] AAkash, K. "Gesture Actuated Robotic Arm", International Journal of Scientific and Research Publications, 2, pp. 1, (2012).

[2] Abidhusain, S., Zamrrud, T., Thimmannagouday, M. and Bheemesh, G. "Flex Sensor Based Robotic Arm Controller Using Micro Controller", Journal of Software Engineering and Applications, 5, pp. 365, (2012).

[3] Colombo, G., Joerg, M., Schreier, R. and Dietz, V. "Treadmill training of paraplegic patients using a robotic orthosis", Journal of rehabilitation research and development, 37(6), pp.693, (2000).

[4] Interlink Electronics, FSR Integration guide. (2000).

[5] Jacob, R., Moshe, B., Moshe, B. and Mircea, A. "A Myosignal-Based Powered Exoskeleton System”, IEEE Transaction on Systems, Man and Cybernetics, 31, pp. 210, (2001).

[6] Kazuo, K., Takakazu, T. and Toshio, F. "Neuro-Fuzzy Control of a Robotic Exoskeleton with EMG Signals", IEEE Transaction on Fuzzy Systems, 12, pp. 481, (2004).

[7] Mosher, Ralph S. Handyman to hardiman. No. 670088. SAE Technical Paper, (1967).

[8] Raju, R. S., Sharang, S. D., Kajal, R. T. and Pawar, S. G. "Mind Controlled Robotic Arm", International Journal of Development Research, 5, pp. 5712, (2015).

[9] Suzuki, K., Mito, G., Kawamoto, H., Hasegawa, Y. and Sankai, Y. "Intention-based walking support for paraplegia patients with Robot Suit HAL", Advanced Robotics, 21(12), pp.1441-1469, (2007).

[10] The Authoritative Dictionary of IEEE Standards Terms (IEEE 100) (seventh ed.). Piscataway, New Jersey: IEEE Press, (2000). 